\title{
COLLIMATION IN THE TRANSFER LINES TO THE LHC
}

\author{
H. Burkhardt, B. Goddard, Y. Kadi, V. Kain, W. Weterings CERN, Geneva, Switzerland
}

\begin{abstract}
The intensities foreseen for injection into the LHC are over an order of magnitude above the expected damage levels. The TI 2 and TI 8 transfer lines between the SPS and LHC are each about $2.5 \mathrm{~km}$ long and comprise many magnet families. Despite power supply surveillance and interlocks, failure modes exist which could result in uncontrolled beam loss and serious transfer line or LHC equipment damage. We describe the collimation system in the transfer lines that has been designed to provide passive protection against damage. Results of simulations to develop a conceptual design are presented. The optical and physical installation constraints are described, and the resulting element locations and expected system performance presented, in terms of the phase space coverage, local element temperature rises and the characteristics of the beam transmitted into the LHC.
\end{abstract}

\section{INTRODUCTION}

Beams will be injected from the SPS into the LHC through the two transfer lines, TI 2 and TI 8 [1]. The batches are extracted in $4 / 11$ of an SPS turn or $7.86 \mu$ s. The transfer lines are pulsed and failures leading to local loss of the injected beam cannot completely be excluded. The damage level for fast losses is estimated to be around $2.3 \times 10^{12}$ protons [2]. Reference numbers are summarised in table 1 .

Table 1: Beam parameters for LHC injection.

\begin{tabular}{|l|r|}
\hline Proton energy & $450 \mathrm{GeV} / \mathrm{c}$ \\
Normalized emittance & $\epsilon_{N}=3.5 \mu \mathrm{m}$ \\
Nominal: & \\
Protons per injection & $3.2 \times 10^{13}$ \\
Ultimate: & \\
Protons per injection & $4.9 \times 10^{13}$ \\
\hline
\end{tabular}

Collimation in the transfer lines should provide protection for nominal and ultimate beam intensities. This leads to the design goal for the transfer line collimators to provide a reduction in the peak energy deposition of at least a factor of $4.9 \times 10^{13} / 2.3 \times 10^{12} \approx 20$, to prevent damage by a mis-steered injected beam arriving at the LHC.

\section{TRANSFER LINE COLLIMATION}

The minimal available physical aperture of the LHC ring is estimated at about 7.5 $\sigma$. It is planned to set the primary LHC collimators to $5.7 \sigma$ at injection and the secondary collimators to $6.7 \sigma$. This reduces the tertiary halo of the circulating beams to below the quench level at physical apertures (on average about $10 \sigma$ ) [3]. However, wrongly injected beams could do damage before they even arrive at the collimation sections, and transfer line collimators will protect the LHC against many of the possible failures, and will also protect critical elements in the lines themselves, such as the injection septum MSI where the aperture is very tight.

The transfer line collimators are only considered as safety devices. Their function is to reduce and dilute the beam intensity sufficiently in a single pass in case of (rare) failures. This requires two-sided collimators at several betatron phases. A setting of about $5 \sigma$ is foreseen, to allows for the expected beam position jitter and some mismatch [4]. Under good conditions, the beam should pass without noticeable loss, since regular beam cleaning will be done by a set of scrapers in the SPS prior to extraction towards the LHC, where a 'shaving' to about $3.5 \sigma$ (corresponding to less than $0.05 \%$ loss for Gaussian beams) is foreseen [5].

\section{DESIGN AND APERTURE CONSIDERATIONS}

The design foresees nine TCDI collimators in each of the transfer lines TI 2 and TI 8. One momentum collimator will limit energy offsets to a level of about $\pm 2 \times 10^{-3}$. Four vertical and four horizontal betatron collimators with flat jaws are arranged to provide complete phase space coverage, to limit the maximum amplitude of oscillation to about $5.4 \sigma$. Ideally, the collimators are located at $0,45,90$ and $135^{\circ}$ phase advance from the injection septum [6].

The maximum amplitudes apply only to one plane $\left(x, x^{\prime}\right.$ or $y, y^{\prime}$ ). Amplitudes up to a factor of $\sqrt{2}$ larger (or up to $7.65 \sigma$, larger than the LHC aperture) are in theory possible for coupled x-y offsets; however, failures leading to such a situation are considered to be extremely unlikely.

Rotation and coupling effects have recently been studied [7]. Due to the geometry of the transfer line TI 8, a $53 \mathrm{mrad}$ mismatch exists between the orientation of the injected beam and the circulating LHC beam, which can couple large $\mathrm{x}$ and $\mathrm{y}$ amplitudes into one or the other plane, leading to increased tails in the particle distribution. Scraping in the SPS will solve this, by removing particles with simultaneous large $\mathrm{x}$ and $\mathrm{y}$ amplitudes.

The optics functions and collimator positions in TI 8 are illustrated in Fig. 1 for betatron collimator section at the end of the line. 


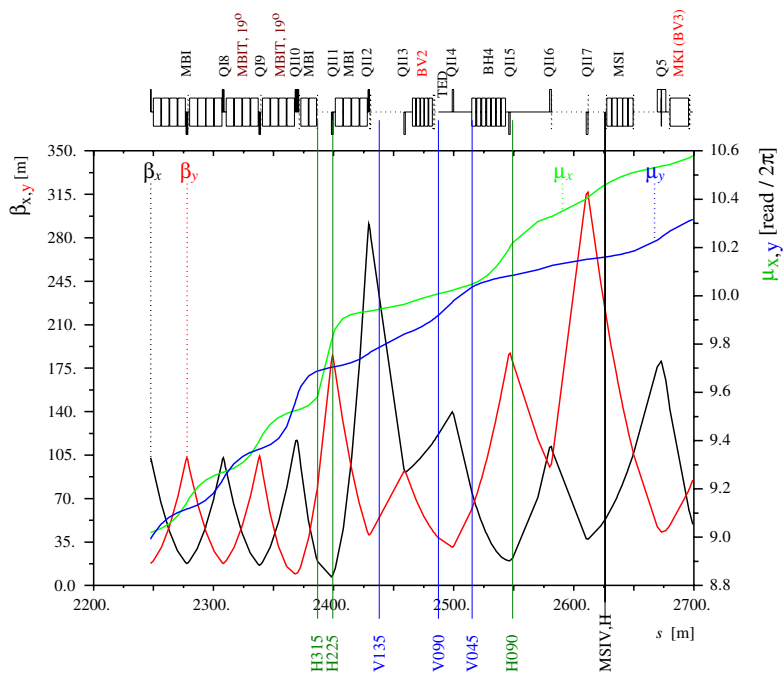

Figure 1: Optics functions and collimator locations at the end of TI 8.

\section{ENERGY DEPOSITION SIMULATIONS}

Preliminary design studies have been completed. The system will be based on $1.2 \mathrm{~m}$ long movable carbon collimators TCDI, similar to the LHC secondary collimators TCS. These provide adequate dilution of the primary beam, with an attenuation of 13.5 and an emittance growth of about a factor of 100 per plane, resulting in an overall dilution efficiency factor of over 100 [8]. In order to protect local elements from shower and scattered particles, the TCDI must be followed by a $0.5 \mathrm{~m} \mathrm{Fe}$ external shield (mask). An example of the geometry of a TCDI and adjacent mask and magnet is shown in Fig. 2.

The detailed technical design studies are in progress for all transfer line collimators, to predict loss patterns and energy deposition in the event of worst-case failures. The $300 \mathrm{~m}$ downstream section of TI 8 was modelled in FLUKA, including all vacuum and magnetic elements, and energy deposition simulations made for full LHC ultimate beam with different impact parameters on the TCDI jaws. The modelling of the line geometry has been made with preprocessing scripts based on the MADX machine layout files, in such a way as to allow straightforward modification and reuse, and also extension to other problems. The magnetic fields in the dipole line elements were included.

Full 3-D energy deposition and temperature maps were produced for TCDIH315 and TCDIH225 as well as for the part of TI 8 up to $30 \mathrm{~m}$ downstream of these collimators.

Fig. 3 shows the temperature profile for the $20 \mathrm{~m}$ downstream of the investigated TCDI for a $1 \sigma$ impact on the collimators TCDIH225 as well as the TCDIH315.

For small impact parameters (in the order of $1 \sigma$ ), a big fraction of the impacting beam is scattered out from the jaw. The simulations show that the adjacent magnet (quadrupole MQID87500 for TCDIH225) is sufficiently protected against the scattered and shower particles by the mask, see Fig. 4.

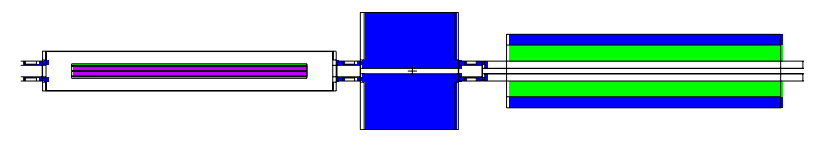

Figure 2: Schematic view of TCDIH225 + mask + MQID87500

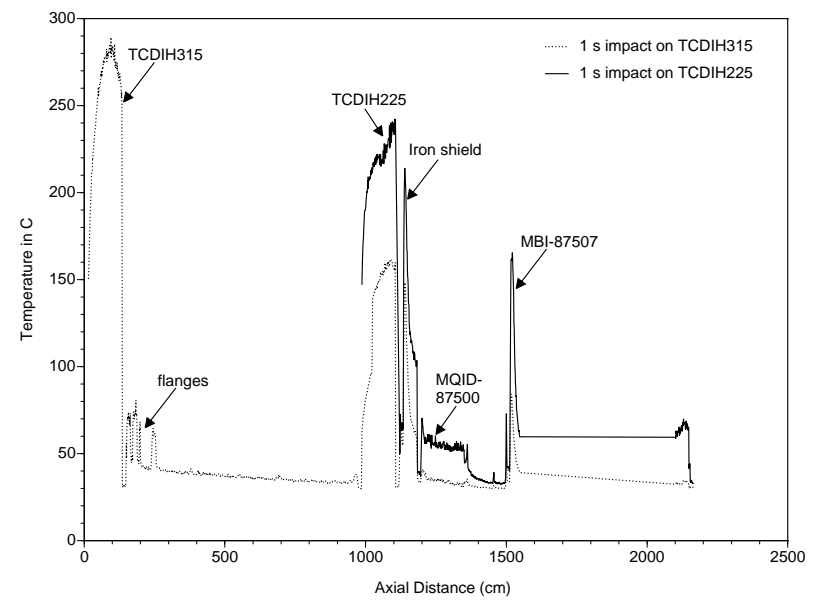

Figure 3: Comparison of the temperatures in the different objects (from TCDIH315 to end of first MBI87507 approximately $20-25 \mathrm{~m}$ ) for a 1 sigma impact on TCDIH315 and on TCDIH225. Note the higher temperatures obtained in the case of a 1 sigma impact on TCDIH225, due to the fact that the beam size is much smaller.

The energy deposition in the first MBI dipole magnet $15 \mathrm{~m}$ downstream of the collimator for a $1 \sigma$ impact on TCDIH225 is shown in Fig. 5 and 6. The shadow effect of the mask (which has an elliptical opening) can be clearly seen in Fig. 5; the peak temperature rise in the MBI iron yoke is $175 \mathrm{~K}$.

Especially for small impact parameters it turned out that the longer $(>5 \mathrm{~m})$ the distance is between collimator and mask, with the mask close to the magnet, the better the protection of the downstream magnet becomes.

In case of larger impact parameters $(>5 \sigma)$ the system TCDI-mask protects the downstream equipment sufficiently well with larger temperature rises in the collimator and the mask (mask temperature rise: $200 \mathrm{~K}$ for $1 \sigma$ impact, $350 \mathrm{~K}$ for $10 \sigma$ on TCDIH225).

These preliminary simulations were done for perfectly collinear collimator jaws, in the absence of alignment errors for any other element. The results of studies including such errors will be used to decide on the possible requirement of angular control for the collimator jaws. 


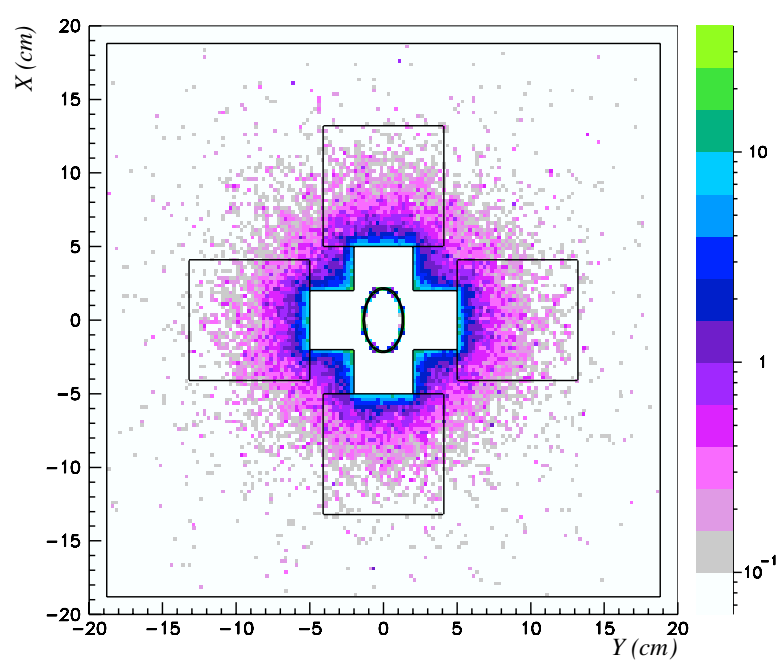

Figure 4: Cross section view of the maximum temperature rise $\Delta T$ in the first part of MQID87500 as a result of a $1 \sigma$ impact on TCDIH225. $\Delta T_{\max }$ is of the order of 10 to $20 \mathrm{~K}$ in the iron part of the magnet.

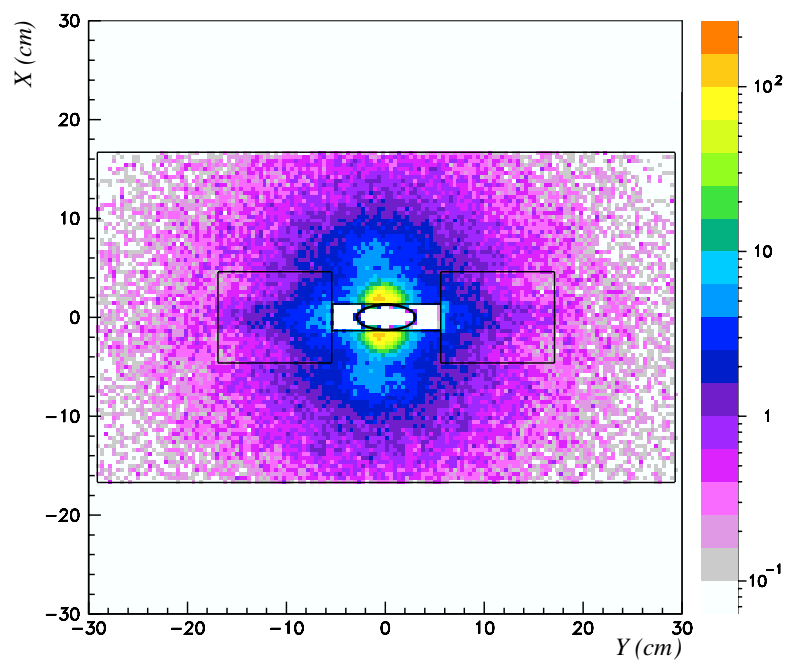

Figure 5: Cross section view of the maximum temperature rise in the first part of MBI87507 as a result of a $1 \sigma$ impact on TCDIH225. $\Delta T_{\max }$ is $175 \mathrm{~K}$ in the iron part of the magnet and somewhat smaller for the connecting flanges.

\section{CONCLUSION}

The transfer line collimators TCDI provide passive protection against damage at injection into the LHC in case of failures. With a set of vertical and horizontal TCDI at 0 , 90,45 and $135^{\circ}$ phase advance from the aperture bottleneck MSI, maximum amplitudes reaching the LHC can be restricted to $5.4 \sigma$. The detailed mechanical design is in progress, based on the TCS design. Simulations show that a graphite jaw of $1.2 \mathrm{~m}$ length gives sufficient beam dilution to protect the LHC in case of failures before injection. For local protection of the line the system is completed with $0.5 \mathrm{~m}$ long external $\mathrm{Fe}$ masks downstream of the collima-

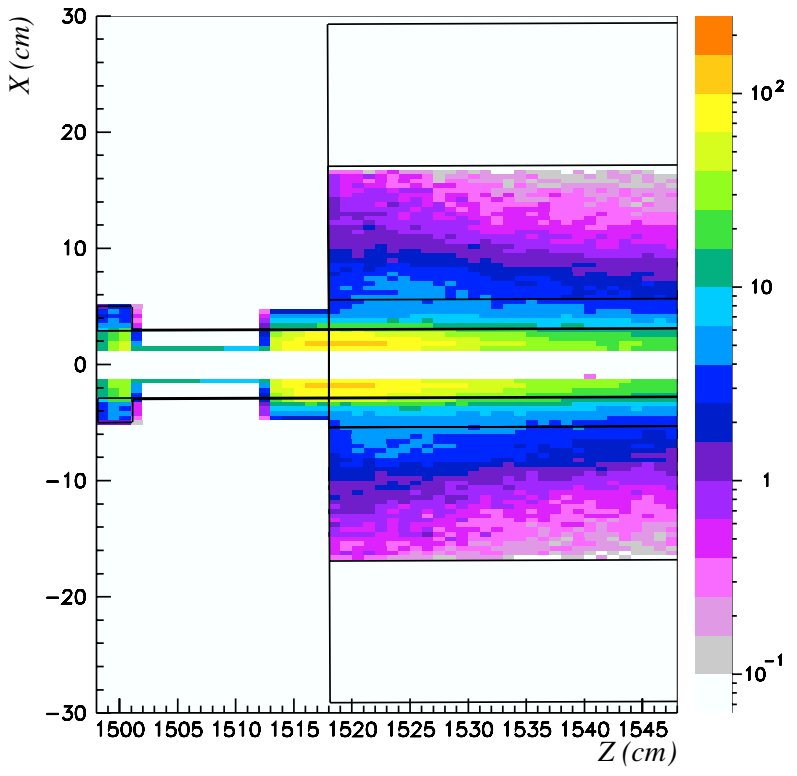

Figure 6: Resulting axial distribution of the maximum temperature rise in the first part of MBI87507 as a result of a $1 \sigma$ impact on TCDIH225.

tors. An energy deposition simulation of the last $300 \mathrm{~m}$ of TI 8 has been set up. Preliminary results show that the system TCDI-mask protects downstream elements from damage in most failure cases; however, with the present layout and load cases, local heating is somewhat above acceptable values, and optimisation studies are continuing.

\section{REFERENCES}

[1] A. Hilaire, V. Mertens, E. Weisse, "Beam Transfer to and Injection into LHC,", LHC Project Report 208 and Proc. EPAC'98, p.2117.

[2] J. Jeanneret, "Collimation schemes and Injection protection Devices in LHC, " Proc. Workshop on LEPSPS performance Chamonix XI, CERN SL/2001-003 (DI) (2001),p.298.

[3] R. Assmann, "An Improved Collimation System for the LHC, ” EPAC'04, Lucerne, Switzerland..

[4] B. Goddard et al., "Aperture and Delivery Precision of the LHC Injection System, ” EPAC'04, Lucerne, Switzerland..

[5] H. Burkhardt, "Collimation Issues at the SPS and in the Transfer lines." Chamonix XIII proceedings, p. 101, CERN AB/2004-014 ADM (2004).

[6] H. Burkhardt, B. Goddard, V. Kain, and W. Weterings, "Function and Concept of TCDI Transfer Line Collimators, ”LHC-TCDI-ES-0001, CERN, Geneva, 2003.

[7] B. Goddard, M. Gyr, V. Kain, R. Risselada, "Geometrical Alignment and Associated Beam Optics Issues of Transfer Lines with Horizontal and Vertical Deflection, " CERN LHC Project Report 719, CERN, 2004.

[8] V. Kain, B. Goddard, Y. Kadi, "Attenuation and Emittance Growth of $450 \mathrm{GeV}$ and $7 \mathrm{TeV}$ Proton Beams in low-Z Absorber Elements, " EPAC'04, Lucerne, Switzerland.. 